\title{
Thermometry of levitated nanoparticles in a hybrid electro-optical trap
}

\author{
E B Aranas, P Z G Fonseca, P F Barker \\ Department of Physics and Astronomy, University College London, Gower Street, London WC1E \\ 6BT, United Kingdom
}

\section{T S Monteiro}

\begin{abstract}
.
There have been recent rapid developments in stable trapping of levitated nanoparticles in high vacuum. Cooling of nanoparticles, from phonon occupancies of $10^{7}$ down to $\simeq 100-1000$ phonons, have already been achieved by several groups. Prospects for quantum ground-state cooling seem extremely promising. Cavity-cooling without added stabilisation by feedback cooling remains challenging, but trapping at high vacuum in a cavity is now possible through the addition of a Paul trap. However, the Paul trap has been found to qualitatively modify the cavity output spectrum, with the latter acquiring an atypical "split sideband" structure, of different form from the displacement spectrum, and which depends on $N$, the optical well at which the particle localises. In the present work we investigate the $N$-dependence of the dynamics, in particular with respect to thermometry: we show that in strong cooling regions $N \gtrsim 100$, the temperature may still be reliably inferred from the cavity output spectra. We also explain the $N$-dependence of the mechanical frequencies and optomechanical coupling showing that these may be accurately estimated. We present a simple "fast cavity" model for the cavity output and test all our findings against full numerical solutions of the nonlinear stochastic equations of motion for the system.
\end{abstract}




\section{Introduction: Levitated Optomechanics}

Much experimental effort is currently being invested in the development of optomechanical set-ups aimed at cooling levitated nanoparticles, from room temperatures down to the quantum regime [1]. Quantum levitated optomechanics offers the prospect of mesoscopic and even macroscopic quantum oscillators with extremely high quality factors and largely decoupled from environmental heating and decoherence. Achieving a high $Q$ - while maintaining low mechanical frequencies $(\sim 100 \mathrm{kHz})$ and comparatively high masses $\left(\sim 10^{7}-10^{10} \mathrm{amu}\right)$ - would make levitated schemes useful for cat-state preparation [2], matter-wave interferometry [3], tests of collapse theories [4, 5], and ultra-sensitive short-range force sensing [6].

In a cryogenic environment, $\mathrm{MHz}-\mathrm{GHz}$ oscillators have reached the ground state at $25 \mathrm{mK}$ [7] or even higher [8,9] in clamped set-ups. However, $\mu \mathrm{K}$ temperatures are needed to attain phonon occupancies of $\bar{n}_{\mathrm{b}} \lesssim 1$ in levitated nanospheres, and cooling from room temperatures is required in most proposed set-ups. Ultra-high vacuum pressures are also needed since ambient gas represents a major source of heating. Although several alternative schemes were proposed in $2010[10,11,12]$ ground state-cooling has not yet been achieved in any levitated system. The key challenge lies in keeping the particle stably trapped while reducing ambient pressures down to ultra-high vacuum. A particle loss mechanism near $\sim 1$ mbar has been identified $[13,14,15]$. While not fully understood, the dependence of particle loss on pressure or laser intensity has been investigated by several groups $[15,16,17]$ in a range of different optical traps.

Active cooling with optical feedback provides one effective means to stabilise the nanoparticle and trapping at pressures down to $\sim 10^{-6}-10^{-8}$ mbar has been achieved. MilliKelvin and submilliKelvin temperatures $[18,19,20,21]$ have been demonstrated and in [21], a phonon occupancy of $\bar{n}_{\mathrm{b}} \simeq 63$ was measured. Although extremely promising, further progress with this approach hinges on overcoming the technical challenge of measurement noise-limited feedback cooling at low $\bar{n}_{\mathrm{b}}[21]$.

Progress towards the usual optomechanical technique of red-sideband cooling in an optical cavity has proved slower, since passive cooling methods cannot sufficiently stabilise the nanoparticle motion as pressures are reduced past the $\sim 1$ mbar bottleneck. In [17], loading of a particle into a cavity already at high vacuum by means of a mobile fiber and lens trap was successfully demonstrated with the aid of feedback. Recently, in [22, 23], a hybrid trap set-up comprising a Paul trap within an optical cavity was shown to trap nanoparticles for indefinite times at high vacuum and without any additional stabilisation.

More broadly, the field of levitated optomechanics continues to experience rapid new development: sympathetic cooling of the centre-of-mass motion by cold atoms has been proposed as an alternative means of achieving ground-state cooling [25]; there is now considerable interest in investigation of rotational modes of aspherical nanoparticles [26]. An interesting new development is the study of torsional modes of motion in levitated nanodiamonds with spin degrees of freedom [27]. Trapping with a type of Paul trap has found an interesting new direction in [28] where electron spin resonance was demonstrated with a levitated nanodiamond. 

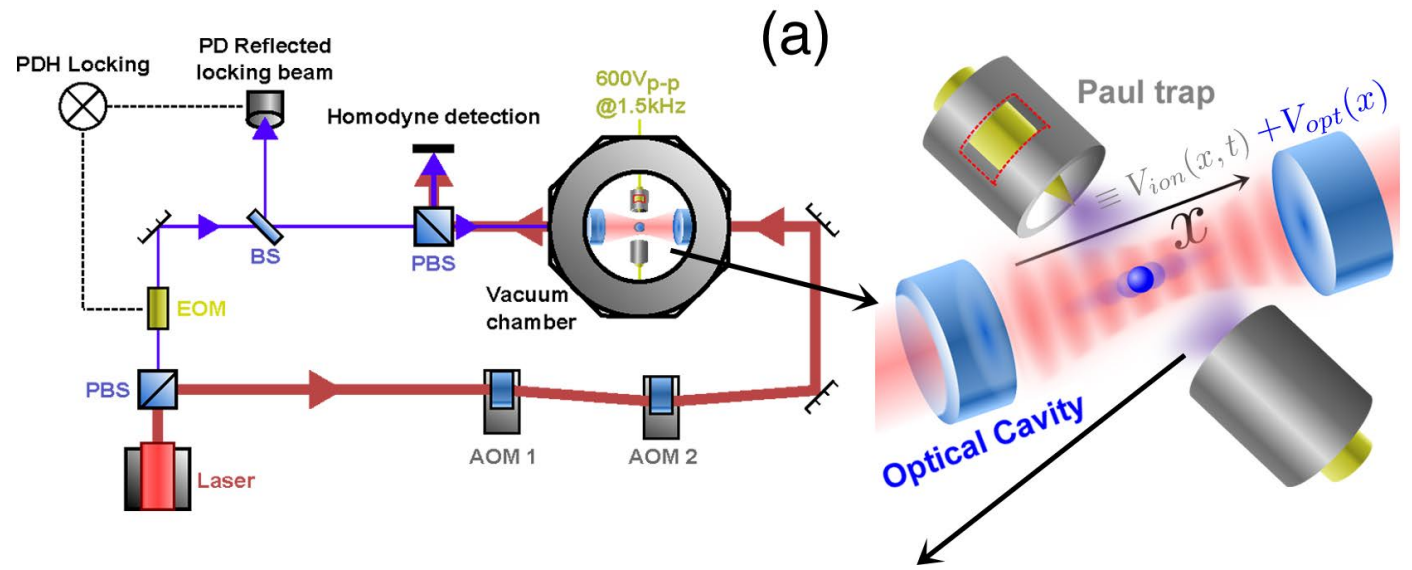

(b)

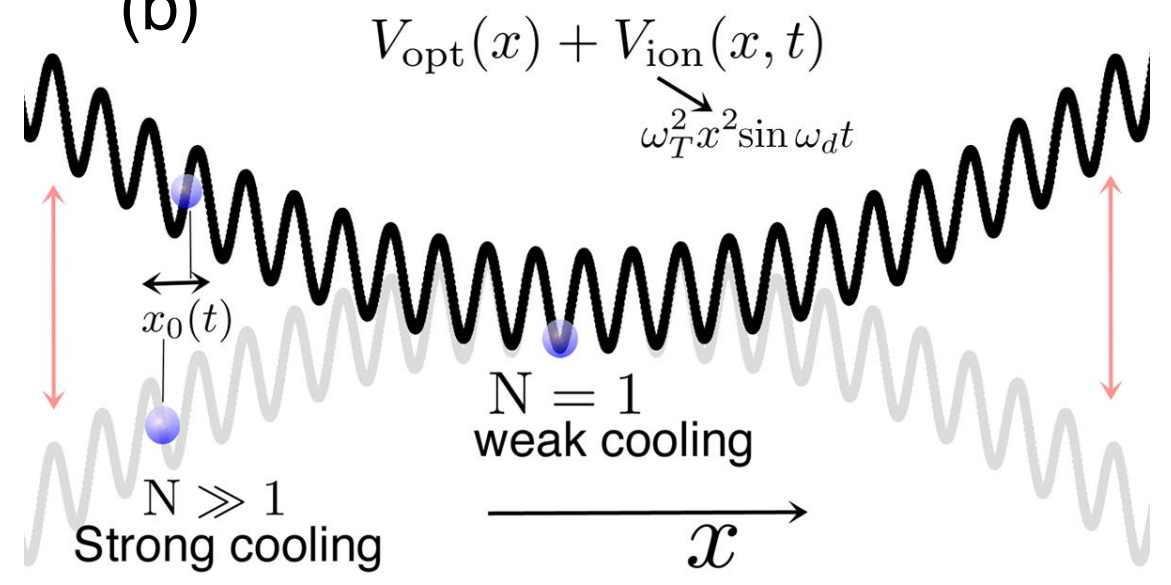

Figure 1. (a) A hybrid electro-optical trap comprises a Paul trap inside an optical cavity. The whole set-up is inside a vacuum chamber. The Paul trap stabilises the nanoparticle held in the combined potentials due to the optical standing wave of the cavity $V_{\text {opt }}$ and ion trap $V_{\text {ion }}$ while the background gas is pumped out. (b) Axial dynamics: a nanoparticle trapped in a single optical well of the optical potential of the cavity oscillates with a frequency $\omega_{M}$ about an equilibrium point $x_{0}$. However, because of the oscillating ion trap field, the position of the equilibrium point itself oscillates slowly with time $x_{0} \equiv x_{0}(t)=X_{\mathrm{d}} \sin \omega_{\mathrm{d}} t$. Linearised analysis is still possible since $\omega_{\mathrm{d}} \ll \omega_{\mathrm{M}}$, so these motions are separable. The dynamics depend on which well $N$ the particle is captured, since the amplitude of the oscillation in the equlibrium point, $X_{\mathrm{d}} \propto N$, depends on $N$. Small $N \simeq 1$ corresponds to a negligible effect on $x_{0}$ and weak cooling. In contrast, higher $N$ corresponds to a larger amplitude oscillation in $x_{0}$ and strong cooling. Another effect of the $x_{0}(t)$ oscillation is to slightly modulate the mechanical frequency $\omega_{\mathrm{M}}(t) \simeq \bar{\omega}_{\mathrm{M}}+2 \omega_{2} \cos 2 \omega_{\mathrm{d}} t$, as well as the optomechanical coupling $g(t)=2 \bar{g} \sin \omega_{\mathrm{d}} t$. As a result, the intracavity spectrum $S_{\text {yy }}(\omega)$ has a very different shape from the mechanical displacement spectrum $S_{\mathrm{xx}}(\omega)$. In the present work we investigate the $N$-dependence of the mechanical frequency and the optomechanical cooling. A key motivation is to examine the usual assumptions which underline optomechanical thermometry, and whether the temperature of a particle in this hybrid trap may be accurately inferred from the sidebands of the cavity output spectrum.

While certain technical challenges remain, the way to ground-state cooling with an optical cavity now seems open, either with or without feedback. There are a range of strong motivating factors: a Fabry-Perot optical cavity provides an exquisitely sensitive means of sensing displacement, exploited recently for the detection of gravitational waves in LIGO [29] where a sensitivity of $10^{-18}$ 
$\mathrm{m}$ was achieved. Importantly, levitation in a cavity allows a non-trivial two-way dynamics between the nanoparticle motion and the cavity mode. This allows immediate access to quantum effects arising from the zero-temperature optical field in the cavity. Optical trapping, whether in FabryPerot cavities or optical tweezers is a result of the dipole optical force acting on a dielectric particle, which implies a potential $V(x)=-\frac{1}{4}\left|\operatorname{Re} \alpha_{\text {ind }}\right| E^{2}(x)$ that depends on the particle polarisability $\alpha_{\text {ind }}$ as well as electric field $E$. However, interaction with the cavity standing wave field $E(x) \propto \cos k x$ represents more than a simple trapping field; the particle shifts the cavity resonant frequency in a position-dependent way, introducing a back-action between the particle motion and the cavity mode degrees of freedom. The net effect is a change in the effective cavity length by the levitated particle, leading to dynamics analogous to the usual clamped cavity optomechanics case where one effectively has a two coupled oscillator system. For clamped set-ups in the quantum regime, this coupling has already allowed interesting demonstrations of quantum back-action effects [30, 31] arising from quantum correlations between incoming cavity noise and the intracavity field.

It is therefore of particular interest to understand the dynamics of the cavity in a Paul trap set-up with no feedback [22, 23] which most closely approximates the usual scenario of cavity optomechanics, with two linearly-coupled oscillator modes. The previous studies in $[22,23]$ have shown, however, that the cavity output differs qualitatively from the usual case: the experimentally observed sidebands of the cavity light, detected by homodyne or heterodyne methods, have a very different spectral profile from the displacement spectra. The distinguishing feature of the cavity spectrum $S_{\mathrm{yy}}(\omega)$ are pairs of peaks at $\omega \simeq\left(\omega_{\mathrm{M}} \pm \omega_{\mathrm{d}}\right)$ and at $\omega \simeq-\left(\omega_{\mathrm{M}} \pm \omega_{\mathrm{d}}\right)$, where $\omega_{\mathrm{M}}$ is the mechanical frequency and $\omega_{\mathrm{d}}$ is the drive frequency of the oscillating Paul trap. In contrast, the displacement spectrum $S_{\mathrm{xx}}(\omega)$ remains peaked at $\omega \simeq \pm \omega_{\mathrm{M}}$.

In [24] a theoretical quantum model based on the linearised dynamics was developed to describe these 'split-sideband' spectra. The model considered the effect of slow modulations of the optomechanical coupling $g(t)=2 \bar{g} \sin \omega_{\mathrm{d}} t$ and of the mechanical frequency $\omega_{\mathrm{M}}(t)=$ $\bar{\omega}_{\mathrm{M}}+2 \omega_{2} \cos 2 \omega_{\mathrm{d}} t$. These modulations modify $S_{\mathrm{yy}}(\omega)$ substantially: with increasing $\omega_{2}$, sideband shapes become asymmetric as the amplitudes of the peaks within each pair become unequal. For $\omega_{2} \sim 2 \omega_{\mathrm{d}}$, the $\omega \simeq \pm\left(\bar{\omega}_{\mathrm{M}}+\omega_{\mathrm{d}}\right)$ peaks are fully suppressed. Note that the above is distinct from Stokes/anti-Stokes asymmetry where (when the mechanical oscillator is near the quantum regime) there is asymmetry between $\omega \simeq-\omega_{\mathrm{M}}$ and $\omega \simeq+\omega_{\mathrm{M}}$ sideband features of the cavity output.

In the present work we also investigate the implications of these characteristics for thermometry: a key question is whether the oscillator temperature may still be reliably inferred from this, atypical, cavity output. We also investigate in detail, by means of simple models and comparisons with full numerics, the behaviour of the mechanical frequencies and optomechanical coupling.

In section 2 we review the dynamics of the hybrid trap, and show how the Paul trap modifies $\omega_{\mathrm{M}}, g$ and to some extent also $\Delta$, the detuning of the laser from the cavity resonance, by inducing a well-dependent excursion in the equilibrium position. In section 3 we investigate the shape of the cavity output spectrum relative to the displacement spectrum; we also derive the dependence of $\bar{g}, \bar{\omega}_{\mathrm{M}}$ and $\omega_{2}$ on experimental parameters, in particular the optical well $N$ where the nanoparticle becomes trapped. A key finding, of importance for any potential sensing applications, is that that 


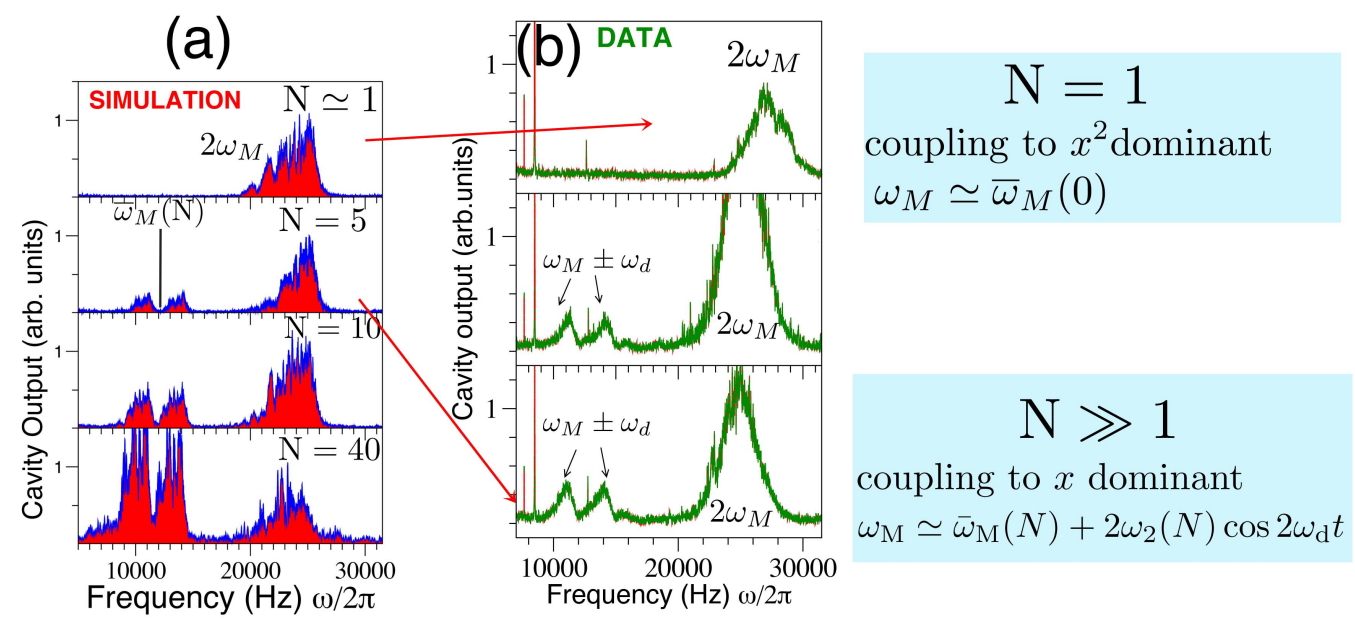

Figure 2. Compares experimental data, against numerical simulations using a stochastic Langevin model, of the cavity output $S_{\text {yout }_{\text {out }}}(\omega)$. For a particle caught in the lowest optical wells $(N \sim 1)$ there is a strong sideband peaked at $\omega \simeq 2 \omega_{\mathrm{M}}$ due to the presence of position-squared $\left(x^{2}\right)$ coupling to the light. In contrast, the effect of the usual optomechanical linear coupling term $g x$ gives rise to a double-peaked structure centered on $\omega \simeq \omega_{\mathrm{M}}$. With increasing $N$, the particle becomes colder and the nonlinear peak becomes relatively weaker, since for a cold particle the $x^{2}$ dependence is strongly suppressed. Note that the linear peak for $N=40$ has larger area than for $N=1$ despite the stronger cooling because of the stronger optomechanical coupling, since $g \propto N$. The mechanical frequency in this system is slightly modulated $\omega_{\mathrm{M}}(t) \simeq \bar{\omega}_{\mathrm{M}}+2 \omega_{2} \cos 2 \omega_{\mathrm{d}} t$. We show here its central value depends on $N$ hence $\bar{\omega}_{\mathrm{M}} \equiv \bar{\omega}_{\mathrm{M}}(N)$. As shown in the figure, the double peaked structures are centered on $\bar{\omega}_{\mathrm{M}}(N)$. Here we present a model which will enable one to read $N$ and hence to infer $\bar{\omega}_{\mathrm{M}}(N)$ and $\omega_{2}(N)$ from the measured cavity output. Data is from [23] at pressure $P \simeq 10^{-2}$ mbar, laser detuning $\Delta=-60 \times 2 \pi \mathrm{KHz}$, input power $P_{\text {in }}=0.07 \mathrm{~mW}$, finesse $F=50,000$, and ion trap frequency $\omega_{\mathrm{T}} \simeq 500 \times 2 \pi \mathrm{Hz}$.

the central frequency depends significantly on $N$, hence $\bar{\omega}_{\mathrm{M}} \equiv \bar{\omega}_{\mathrm{M}}(N)$ and this is investigated in section 3. In section 4 we consider a simple model valid for the fast cavity regime $\kappa \gg \omega_{\mathrm{M}}$ and we show this fast-cavity model can be used to obtain split-sideband asymmetry. In section 5, we analyse thermometry with split-sideband spectra. We show the area under a properly normalised cavity output still reliably gives a temperature consistent with the results obtained from simple period-averaging (i.e., using period-averaged parameters in standard optomechanical expression for cooling rates). In addition to the new analysis in sections 3 and 4, figure 3 and figure 4 represent the main new results. All our analytical findings are verified against nonlinear, stochastic numerics. We summarise and conclude in section 6.

\section{Review: Dynamics of the hybrid Paul-trap in a cavity set-up}

We consider a charged, dielectric nanosphere levitated in a hybrid trap that combines the optical potential from the standing wave in the cavity with the ion trap potential:

$$
\begin{aligned}
V_{\mathrm{TOT}}(x, y, z, t) & =V_{\text {opt }}(x, y, z, t)+V_{\text {ion }}(x, y, z, t) \\
& =\hbar A|a(t)|^{2} \cos ^{2}(k x) \mathcal{F}(y, z)+\frac{1}{2} m \omega_{\mathrm{T}}^{2}\left(x^{2}+y^{2}-2 z^{2}\right) \sin \omega_{\mathrm{d}} t
\end{aligned}
$$


where $|a(t)|^{2}$ represents the (fluctuating) photon number in the cavity, and $x$ is the coordinate

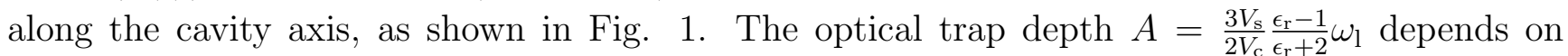
constant parameters: relative dielectric constant $\epsilon_{\mathrm{r}} \simeq 2$ for silica, laser frequency $\omega_{1}$, sphere volume $V_{\mathrm{s}}=\frac{4}{3} \pi R^{3}$, and mode volume $V_{\mathrm{c}}=\pi w^{2} L$ (where $w$ is the beam waist and $L$ is the cavity length). $\mathcal{F}(y, z)=\exp \left(-2\left(y^{2}+z^{2}\right) / w^{2}\right)$ represents the transverse Gaussian beam profile. The axial Paul trap potential $V_{\text {ion }}(x, y=0, z=0, t)=\frac{1}{2} m \omega_{\mathrm{T}}^{2} x^{2} \sin \omega_{\mathrm{d}} t$ represents a harmonic potential with ion trap frequency $\omega_{\mathrm{T}}^{2}=\frac{2 Q V_{0}}{m r_{0}^{2}}$, where $m$ and $Q$ are the mass and charge of the bead, $V_{0}$ is the voltage, and $r_{0}$ is a parameter which characterises the ion trap (see [32] for a review of quadrupole ion traps). In the set-up of [23], $A \simeq 2 \pi \times 26 \mathrm{kHz}, L=1.3 \mathrm{~cm}, w=60 \mu \mathrm{m}, R=200 \mathrm{~nm}, r_{0}=0.5$ $\mathrm{mm}, \omega_{\mathrm{d}}=1500 \mathrm{~Hz}$ and $V_{0}=300 \mathrm{~V}$. In [13] it was found that $R=200 \mathrm{~nm}$ nanoparticles are still reasonably well described by point-dipole approximations, despite their size.

Dipole/gradient forces allow the nanosphere to be captured in one of the optical wells where it oscillates about the antinode $\left(x_{0}=0\right)$ with a mechanical frequency $\omega_{\mathrm{M}} \gg \omega_{\mathrm{d}}$ which is fast relative to the ion trap drive. We label the optical wells by $N$, where $N \in[0: \pm 1000]$ is the representative number of wells spanned by the Paul trap dimension $r_{0}$. In the absence of the Paul trap, $\omega_{\mathrm{M}}$ is insensitive to the well number $N$ the bead is caught in. However, the effect of the ion trap is to periodically pull the charged bead away from the antinode and across the optical well with the drive frequency $\omega_{\mathrm{d}}$. This slow, harmonic excursion in the equilibrium position $x_{0}(t)$, in addition to a fast mechanical oscillation about $x_{0}$, is a characteristic of the hybrid trap dynamics (see figure 1) that not only drives cooling, but also causes well-dependent ( $N$-dependent) optomechanical modulations.

Figure 1 illustrates how the Paul trap removes the $x$-invariance of the different optical wells of the cavity standing wave $V_{\text {opt }}$. The dynamics become well-dependent, with low $N$ associated with a small $x_{0}$ excursion, and a dominant quadratic coupling; while a high- $N$ catch resulting in a large $x_{0}(t)$ and strong cooling. Both regimes were experimentally observed in [23].

In the present paper, we solved the equations of motion in the combined potential of (2). However, cooling dynamics occurs primarily in the axial direction, so in the modelling and analysis of the linearised motions below, we focus on the axial $(x)$ degree of freedom. In the numerical simulations, we always consider the full 3D equations of motion with the addition of a Gaussian noise bath which, in the absence of cooling, yields steady state temperatures of $T \simeq 300 \mathrm{~K}$. Full details were presented in [23], but in the axial direction we solve:

$$
\ddot{x}=-\frac{\hbar k A}{m}|a(t)|^{2} \sin (2 k x) \mathcal{F}(y, z)-\Gamma_{\mathrm{M}} \dot{x}-\omega_{\mathrm{T}}^{2} x \cos \left(\omega_{\mathrm{d}} t\right)-\zeta_{x}(t),
$$

and the noise terms arise from the background bath of gas at room temperature:

$$
\left\langle\zeta_{x}\left(t^{\prime}\right) \zeta_{x}(t)\right\rangle \simeq \Gamma_{\mathrm{M}} 2 n_{B} \frac{\hbar \omega_{\mathrm{M}}}{m} \delta\left(t-t^{\prime}\right)
$$

where $n_{\mathrm{B}}=k_{\mathrm{B}} T /\left(\hbar \omega_{\mathrm{M}}\right)$, with $T_{\mathrm{B}}=300 \mathrm{~K}$ and $k_{\mathrm{B}}$ is Boltzmann's constant. $\zeta_{x}$ and $\eta(t)$ are modelled as Gaussian random noise. For the levitated experimental parameters used here, $\Gamma_{\mathrm{M}} \approx 0.11 \times 10^{4} \mathrm{P} \mathrm{s}^{-1}$ where the pressure $P$ is in mBar. 
For the optical mode, we solve:

$$
\dot{a}=i \Delta a-i \mathcal{E}+i A a \cos ^{2}(k x) \mathcal{F}(y, z)-\frac{\kappa}{2} a-\eta(t) .
$$

Here $\eta(t)$ is assumed to be photon shot noise, where

$$
\left\langle\eta\left(t^{\prime}\right) \eta(t)\right\rangle=\kappa \delta\left(t-t^{\prime}\right)
$$

where $\mathcal{E}$ is the amplitude of the laser driving the cavity with photons of frequency $\omega_{1}$; it is related

to the input power $P_{\text {in }}$ (for a critically coupled cavity) by $\mathcal{E}^{2}=\frac{\kappa}{2} \frac{P_{\text {in }}}{\hbar \omega_{1}}$. For a cavity detuned by $\Delta$ from resonance, this results in a mean cavity photon number $n=\frac{\mathcal{E}^{2}}{\left(\frac{\kappa}{2}\right)^{2}+\Delta^{2}}$. Since the steady state photon number is very large, we note $V_{\text {opt }}=\hbar A|a(t)|^{2} \cos ^{2}(k x) \mathcal{F}(y, z) \simeq \hbar A n \cos ^{2}(k x) \mathcal{F}(y, z)$ so the optical potential is typically nearly independent of time.

The numerical equations are nonlinear as well as stochastic. Crucially, the numerics make no assumptions concerning the behaviour of $\omega_{\mathrm{M}}$, optomechanical coupling strength $g$ or even the modulation in the spring constant (the parameter $\omega_{2}$, discussed below) arising from the excursion in $x_{0}$. All of these, including the excursion in $x_{0}(t)$, emerge from the numerics, once experimental parameters such as laser input power $P_{\text {in }}$, detuning $\Delta$, nanosphere charge $Q$, and well $N$ are set. We note that $Q$ varies between experimental runs, as does $N$ the optical well the particle was captured by; $Q$ and $N$ cannot currently be preset in the experiments and varied unpredictably from one run to the next.

Thus the numerics provide a stringent and independent check on the theoretical analysis based on optomechanical theory and linearised dynamics. Figure 2 shows a comparison between the stochastic numerical simulations of the cavity output and data, for optical capture in low $N$, showing that solving the full nonlinear equations of motion accurately reproduces detailed features of the data. At the lowest $N$, as shown in [23], the cavity spectra are dominated by position squared coupling to the light. This is not investigated in detail here: $x^{2}$ coupling effects are strongly suppressed with increasing $N$, and in the high cooling regimes of $N \gtrsim 100$, linear dynamics predominates. Of more significance is the striking 'twin-peak' sideband structure seen near $\omega \simeq \omega_{\mathrm{M}} \pm \omega_{\mathrm{d}}$. For $N \gtrsim 100$ in fact, these peaks become asymmetric with a ratio $0 \leq r<1$ of the amplitudes of the two peaks. This ratio also depends on $N$. In the following section we analyse these effects from the linearised dynamics, both classical and quantum.

\subsection{Linearised classical dynamics}

Although we do not have to input values of $\omega_{\mathrm{M}}$ or $g$ into the numerics, one may obtain good analytical estimates from the linearised dynamics, given values for the Paul trap drive frequency $\omega_{\mathrm{d}}$, voltage $V_{0}$, detuning $\Delta$, input power $P_{\mathrm{in}}$, as well as nanosphere charge $Q$ and intracavity photon number $n$. For convenience, we shift the origin to the antinode of the trapping well, $x=x_{N}=N \frac{\lambda}{2}$ instead of the origin of the Paul trap at $x=0$. We then adopt the usual procedure of linearising 
about small displacements in position $x(t) \rightarrow x_{0}+x(t)$ and optical field $a(t) \rightarrow \bar{\alpha}+a(t)$. Hence $n=|\bar{\alpha}|^{2}$ is the mean number of photons in the cavity.

In the case of the hybrid trap, however, $x_{0}$ is time-dependent. From the zeroth order dynamics, we can show [23]:

$$
\sin 2 k x_{0}(t)=2 k X_{\mathrm{d}} \sin \omega_{\mathrm{d}} t, \text { where } 2 k X_{\mathrm{d}}=-\frac{\omega_{\mathrm{T}}^{2}}{\bar{\omega}_{\mathrm{M}}^{2}} 2 \pi N .
$$

From the first order dynamics we can show [23]:

$$
\bar{\omega}_{\mathrm{M}}^{2}(0)=\frac{2 \hbar k^{2} A|\bar{\alpha}|^{2}}{m} .
$$

and that with the $x_{0}(t)$ excursion, the optomechanical parameters are given by:

$$
\begin{aligned}
& g\left(x_{0}\right)=k A \bar{\alpha} \sin 2 k x_{0}(t) \\
& \Delta\left(x_{0}\right)=\Delta+A \cos ^{2} k x_{0}(t) \\
& \omega_{\mathrm{M}}^{2}\left(x_{0}\right)=\bar{\omega}_{\mathrm{M}}^{2}(0) \cos 2 k x_{0}(t) .
\end{aligned}
$$

Since $\omega_{\mathrm{d}} \ll \omega_{\mathrm{M}}$, however, $x_{0}(t)$ varies slowly on the time-scale of a mechanical oscillation allowing separate consideration of the motions; thus we can use the instantaneous values of $x_{0}(t)$, and substitute values from (6) into the above equations.

\subsection{Linearised quantum dynamics}

The quantum dynamics were investigated in [24] by considering modulations in the optomechanical coupling $g(t)=2 \bar{g} \sin \omega_{\mathrm{d}} t$ and mechanical frequency $\omega_{\mathrm{M}}(t)=\bar{\omega}_{\mathrm{M}}+2 \omega_{2} \cos 2 \omega_{\mathrm{d}} t$. In the present section, we briefly discuss the dependence of a general optomechanical system on these types of modulations: the behaviour is generic, in the sense that the resulting split-sideband spectral structure could be obtained in any set-up where the spring constant and $g$ are both modulated. In the next section we consider how $\bar{g}, \bar{\omega}_{\mathrm{M}}$ and $\omega_{2}$ vary in the specific case of the hybrid trap.

In the quantum regime, very analogous equations are obtained by considering small quantum fluctuations about equilibrium classical values $\hat{\mathrm{x}}(t) \rightarrow x_{0}+\hat{\mathrm{x}}(t)$ and optical field $\hat{a}(t) \rightarrow \bar{\alpha}+\hat{a}(t)$ The quantum dynamics is given by the linearised quantum Hamiltonian:

$$
\hat{H} / \hbar=\Delta \hat{a}^{\dagger} \hat{a}+\omega_{\mathrm{M}}\left(\hat{\mathrm{p}}^{2}+\hat{\mathrm{x}}^{2}\right)+g\left(\hat{a}^{\dagger}+\hat{a}\right) \hat{\mathrm{x}}
$$

where $\hat{a}^{\dagger}, \hat{a}$ are creation and annihilation operators for small fluctuations in the cavity mode about its equilibrium value $\bar{\alpha}$, while $\hat{\mathrm{x}} \equiv \hat{b}+\hat{b}^{\dagger}$ in appropriately scaled units. The corresponding equations of motion may be solved in Fourier space and one obtains the well-known expression:

$$
\hat{\mathrm{y}}(\omega)=g \eta(\omega) \cdot \hat{\mathrm{x}}(\omega)+\sqrt{\kappa} \hat{Y}_{\text {in }}(\omega),
$$

where $\hat{y}(\omega)=\hat{a}^{\dagger}(\omega)+\hat{a}(\omega)$. This expression relates the intra-cavity field amplitude quadrature to the particle motion; an analogous expression can be written for the phase quadrature of the 
optical field (note that for $\Delta=0$, only the phase quadrature couples appreciably to the motion; however we are considering non-zero $\Delta$ in the present work).

However, if one considers modulated parameters $g(t)=2 \bar{g} \sin \omega_{\mathrm{d}} t$ and mechanical frequency $\omega_{\mathrm{M}}(t)=\bar{\omega}_{\mathrm{M}}+2 \omega_{2} \cos 2 \omega_{\mathrm{d}} t$, which are substituted into (11) followed by solution of the corresponding Heisenberg's equations of motion, including dissipative terms, one now obtains instead:

$$
\hat{\mathrm{y}}(\omega)=\bar{g} \eta(\omega) \cdot\left[\hat{\mathrm{x}}\left(\omega+\omega_{\mathrm{d}}\right)-\hat{\mathrm{x}}\left(\omega-\omega_{\mathrm{d}}\right)\right]+\sqrt{\kappa} \hat{Y}_{\text {in }}(\omega) .
$$

We define the functions $\eta(\omega)=\chi_{\mathrm{O}}(\omega)-\chi_{\mathrm{O}}^{*}(-\omega)$ and $\mu(\omega)=\chi_{\mathrm{M}}(\omega)-\chi_{\mathrm{M}}^{*}(-\omega)$, given in terms of the usual optical susceptibility $\chi_{\mathrm{O}}(\omega)=\left[-i(\omega+\Delta)+\frac{\kappa}{2}\right]^{-1}$ and mechanical susceptibility $\chi_{\mathrm{M}}(\omega)=\left[-i\left(\omega-\omega_{\mathrm{M}}\right)+\frac{\Gamma_{\mathrm{M}}}{2}\right]^{-1}$ respectively.

One may also obtain the quantum displacement spectrum:

$$
\begin{aligned}
& \hat{\mathrm{x}}(\omega)=\bar{g} \mu(\omega) \cdot\left(\hat{\mathrm{y}}\left(\omega+\omega_{\mathrm{d}}\right)-\hat{\mathrm{y}}\left(\omega-\omega_{\mathrm{d}}\right)\right)+\sqrt{\Gamma_{\mathrm{M}}} \hat{X}_{\mathrm{th}}(\omega) \\
& -i \omega_{2} \chi_{\mathrm{M}}(\omega)\left\{\hat{b}\left(\omega+2 \omega_{\mathrm{d}}\right)+\hat{b}\left(\omega-2 \omega_{\mathrm{d}}\right)\right\}+i \omega_{2} \chi_{\mathrm{M}}^{*}(-\omega)\left\{\hat{b}^{\dagger}\left(\omega+2 \omega_{\mathrm{d}}\right)+\hat{b}^{\dagger}\left(\omega-2 \omega_{\mathrm{d}}\right)\right\},
\end{aligned}
$$

The origin of the 'twin peaks' behavior is clear from (13): the $g$-excursion couples the optical field to shifted displacement $\hat{\mathrm{X}}^{ \pm}(\omega)=\hat{\mathrm{x}}\left(\omega+\omega_{\mathrm{d}}\right)-\hat{\mathrm{x}}\left(\omega-\omega_{\mathrm{d}}\right)$. Conversely, it is also apparent from (15) that the $\omega_{2}$ modulation causes $\hat{x}(\omega)$ to develop sidebands at $\bar{\omega}_{M} \pm 2 \omega_{\mathrm{d}}$. The fact that the cavity spectrum is related to a frequency-shifted displacement (and vice-versa) makes this system different from the canonical optomechanical system where $\hat{y}(\omega)$ is simply related to $\hat{x}(\omega)$.

By means of iterative substitution of (14) in (13) (and vice-versa) an approximate solution to the cavity output spectrum may be obtained, which was found to give good agreement with numerics for moderate values of $\omega_{2}$. It was found that the asymmetry which develops between peak heights as $\omega_{2}$ increases, is due to interference effects. Details are in [24] but from these solutions, an expression for ratio $r$ of the heights of the two peaks was obtained:

$$
r \approx\left(2 \omega_{\mathrm{d}}-\omega_{2}\right)^{2} /\left(2 \omega_{\mathrm{d}}+\omega_{2}\right)^{2}
$$

While in [24] these behaviors were investigated for an arbitrary optomechanical system, the corresponding modulations also arise naturally for an optically-trapped particle with a sinusoidal modulation $x_{0}(t)$ of the equilibrium position of the oscillator. Taking $g\left(x_{0}\right)$ and $\omega_{\mathrm{M}}\left(x_{0}\right)$ from $(8)$ to (10) it is shown in the next section that they lead to similar modulations of the mechanical frequency $\omega_{\mathrm{M}}(t) \simeq \bar{\omega}_{\mathrm{M}}+2 \omega_{2} \cos 2 \omega_{\mathrm{d}} t$ as well as $g(t)=2 \bar{g} \sin \omega_{\mathrm{d}} t$. The $N$-dependence specific to the hybrid trap emerges only when we substitute (6) in (8) to (10). Comparisons with numerics found that $r$ is reasonably approximated by Eq. 15 provided $\omega_{2} \lesssim \omega_{d}$. For larger $\omega_{2}$, the ratio $r$ must be estimated numerically.

\section{Results: $N$-dependence of experimental parameters}

Because of the $x_{0}(t)$ excursion, the hybrid trap exhibits atypical cavity output spectra. For $N=1$ (figure 2), the modulation of $x_{0}$ is minimal and does not change the optomechanical parameters 

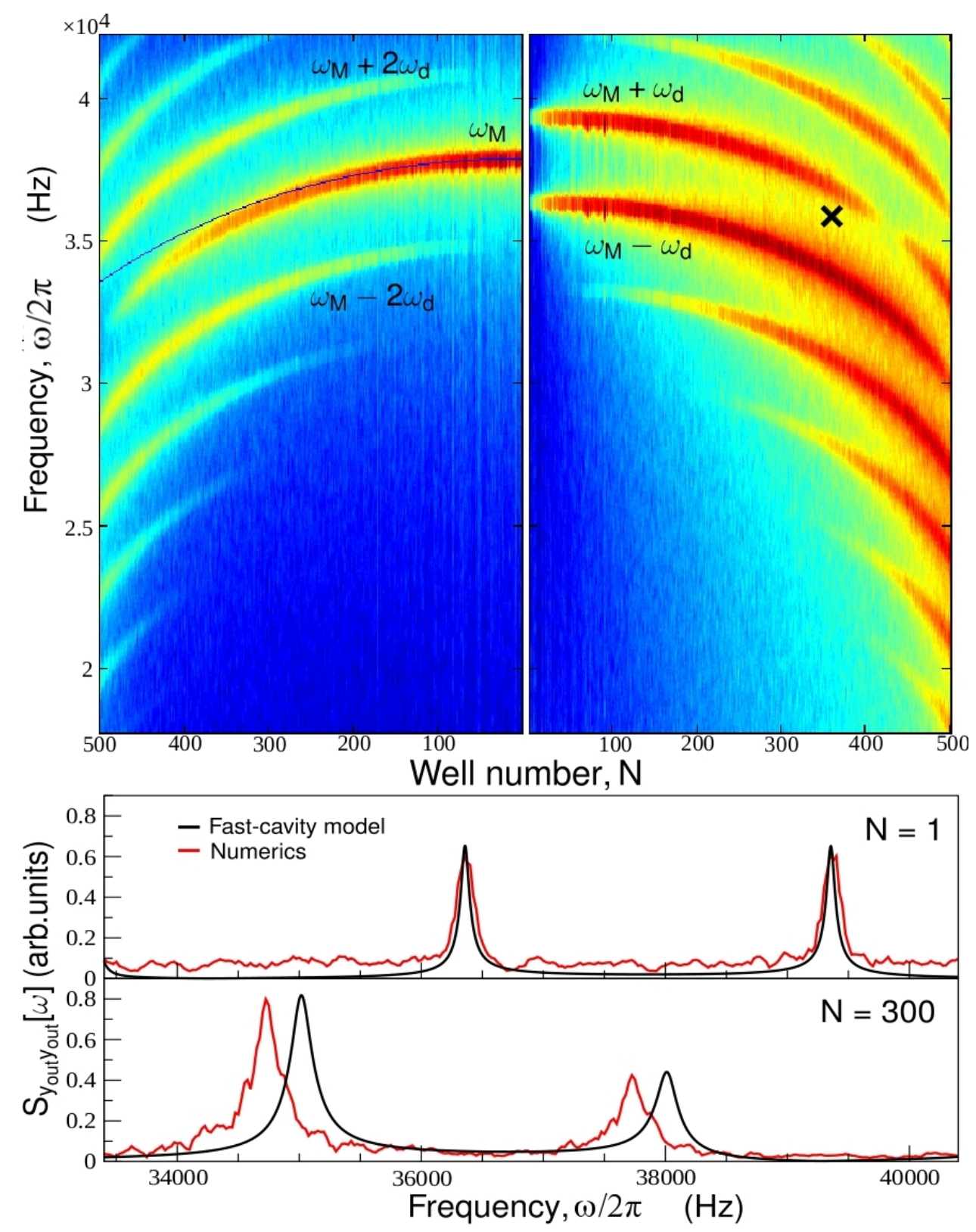

Figure 3. (a) Colormaps showing the displacement spectra (left panel $S_{\mathrm{xx}}(\omega)$ ) and cavity output (right panel, $\left.S_{\mathrm{y}_{\text {out }} \text { out }}(\omega)\right)$ for $N \in[1: 500]$, obtained by numerical solution of nonlinear coupled Langevin equations. Colors indicate the noise power at each frequency. $S_{\mathrm{xx}}(\omega)$ is peaked at $\omega=\bar{\omega}_{\mathrm{M}}(N)$ which decreases with increasing $N$. The blue line represents the analytical Paul trap-shifted $\bar{\omega}_{\mathrm{M}}(N)$ and shows good agreement with the numerics up to $N \approx 400$. Additional weaker sidebands at $\omega \simeq \omega_{\mathrm{M}} \pm 2 \omega_{\mathrm{d}}$ are also seen at higher $N$. In contrast, $S_{\text {yout }_{\text {out }}}(\omega)$ is mainly peaked at $\omega \simeq \bar{\omega}_{\mathrm{M}} \pm \omega_{\mathrm{d}}$; For low $N$, a 'twin peaks' structure is seen; with increasing $N$ an asymmetric pair develops as the $\omega \simeq \bar{\omega}_{M}+\omega_{\mathrm{d}}$ peak is increasingly suppressed. We compute the asymmetry ratio using a fast-cavity model which predicts complete cancellation of $\bar{\omega}_{\mathrm{M}}+\omega_{\mathrm{d}}$ peak at $N \approx 362$ (marked by $\times$ ). Higher-order sidebands at $\bar{\omega}_{\mathrm{M}}(N) \pm n \omega_{\mathrm{d}}$ for odd $n$ also appear as the frequency modulation becomes stronger. (b) Shows individual spectra for $N=1$ (twin peaks) and $N=300$ (asymmetric peaks). Numerics $\equiv$ red and analytical fits $\equiv$ black. Input power $P_{\text {in }}=0.6 \mathrm{~mW}$, pressure $P=5.4 \times 10^{-2} \mathrm{mbar}$, laser detuning $\Delta=-100 \times 2 \pi \mathrm{kHz}$, and ion trap frequency $\omega_{\mathrm{T}} \simeq 630 \times 2 \pi \mathrm{Hz}$. 
significantly (although spectra here are strongly affected by position-squared coupling). For higher $N \gtrsim 100$ only the linear coupling is significant; however, instead of the usual single peak at $\omega=\omega_{\mathrm{M}}$ there is a pair of sideband-peaks at $\omega_{\mathrm{M}} \pm \omega_{\mathrm{d}}$.

Substitution of (6) in (8) gives the optomechanical coupling in terms of $N$ :

$$
g(N, t)=2 \bar{g}(N) \sin \omega_{\mathrm{d}} t
$$

which oscillates about an $N$-dependent mean:

$$
\bar{g}(N)=\frac{\omega_{\mathrm{T}}^{2}}{\bar{\omega}_{\mathrm{M}}(0)^{2}} \pi k A|\bar{\alpha}| N
$$

Another consequence of a high- $N$ catch is the Paul trap pulls the nanosphere away from the antinode and towards the linear region of the optical well, thereby modulating $\omega_{\mathrm{M}}$ as well. We square (6) and (10), add them, and then solve for $\omega_{\mathrm{M}}(t)$ :

$$
\omega_{\mathrm{M}}(t)=\bar{\omega}_{\mathrm{M}}(0)\left(1-\left(2 k X_{\mathrm{d}}\right)^{2} \sin ^{2} \omega_{\mathrm{d}} t\right)^{1 / 4} .
$$

Expanding up to second order in $\zeta$, we obtain:

$$
\omega_{\mathrm{M}}(N, t) \approx \bar{\omega}_{\mathrm{M}}(N)+2 \omega_{2}(N) \cos 2 \omega_{\mathrm{d}} t
$$

where:

$$
\begin{aligned}
& \bar{\omega}_{\mathrm{M}}(N) \approx\left(1-\zeta N^{2}-\frac{9}{4} \zeta^{2} N^{4}\right) \bar{\omega}_{\mathrm{M}}(0), \text { and } \\
& \omega_{2}(N) \approx \frac{1}{2}\left(\zeta N^{2}+3 \zeta^{2} N^{4}\right) \bar{\omega}_{\mathrm{M}}(0) .
\end{aligned}
$$

with $\zeta=\frac{\pi^{2}}{2} \frac{\omega_{\mathrm{T}}^{4}}{\bar{\omega}_{\mathrm{M}}^{4}(0)}$. Equation (19) underlines the non-trivial, two-way interaction between the cavity and Paul trap field a hybrid trap: the mechanical frequency is shifted and modulated by the Paul trap, while the secular frequencies of the Paul trap acquire a "cavity-shift" from the optical field [23]. We verify this through numerics where we solve nonlinear coupled Langevin equations using stochastic Runge-Kutta algorithm. We run the simulation for the same parameters as the strong cooling data in figure 4 of [23], and present the cavity spectra for $N=1: 500$ in figure 3a. The right panel clearly shows the twin peaks at low $N$, with progressive suppression of $\omega \simeq \bar{\omega}_{\mathrm{M}}+\omega_{\mathrm{d}}$. Note that as the map is a log plot, the weak but more complex structure at high $N$ is emphasized.

The mechanical frequency shifts by about 10\%, in agreement with (20). For comparison we overlay the value of $\bar{\omega}_{\mathrm{M}}(N)$ obtained from (20), showing reasonable agreement up to about $N \lesssim 400$. Obtaining agreement for higher $N \gtrsim 400$ becomes increasingly challenging. One potential source of the discrepancy may be because, in using (7) to calculate $\bar{\omega}_{\mathrm{M}},|\bar{\alpha}|^{2}$ has to be appropriately corrected as a result of a modified detuning (9). It also has an $N$ dependence since $\Delta(N) \approx \Delta+A-2 A \zeta N^{2}$. The modulation of $\Delta$ due to the change in $x_{0}(t)$ is usually a minor effect, provided $\Delta \gg A$ and $\kappa \gtrsim|\Delta|$. This approximation is tested here by the comparisons with the numerics which involve no linearisation: in the numerics the effective detuning, as well as $\omega_{\mathrm{M}}$ and $g$, are emergent properties, not input parameters.

It is important to note that the $\bar{\omega}_{\mathrm{M}}(N)$ shifts are quite different from the usual optical spring effect seen in standard optomechanics and can be significantly larger. As for the modulation of 


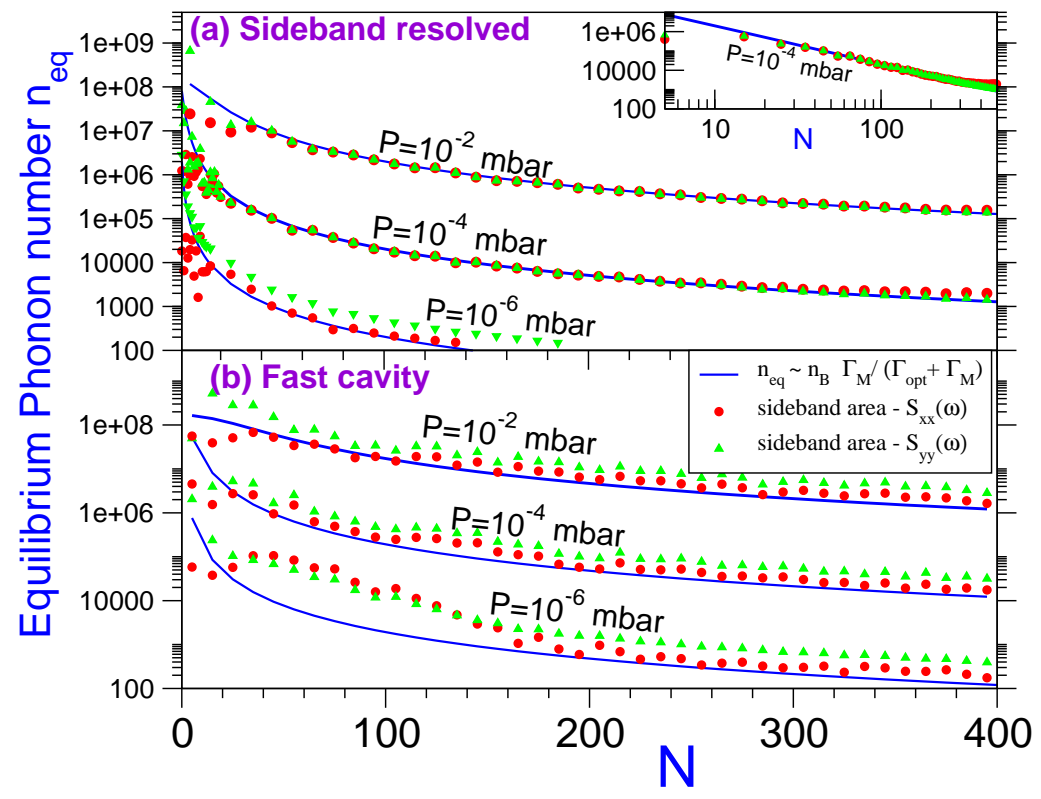

Figure 4. Thermometry in hybrid traps: compares final (steady state) phonon numbers inferred from the area under the sidebands of the normalised cavity output $S_{\mathrm{y}_{\text {out }} \text { out }}(\omega) /\left(\bar{g}^{2}|\eta(\omega)|^{2}\right)$ (green triangles) with those calculated directly from the displacement spectrum $S_{\mathrm{xx}}(\omega)$ (red circles). Results are given as a function of $N$, the optical well where the nanoparticle is trapped. The blue line represents the values using the standard optomechanical cooling formula with a periodaveraged coupling $\bar{g}(N)$. Agreement between the three methods is excellent, except the very lowest few capture wells $N \lesssim 10$ where there is a strong contribution from the position-squared coupling term. In this case, the cavity output estimate (which integrates over both linear and nonlinear sidebands is much larger than the value estimated from $S_{\mathrm{xx}}(\omega)$. The inset shows the final phonon occupancy scales as $N^{-2}$. Upper panel parameters are for a high-finesse cavity $\mathcal{F}=200,000$ which can yield ground state cooling for capture in high wells $N \gtrsim 300-500$ for pressures $P=10^{-6}-10^{-7}$ mbar; input power $P_{\text {in }}=0.6 \mathrm{~mW}$, laser detuning $\Delta=-70 \times 2 \pi \mathrm{kHz}$, ion trap frequency $\omega_{\mathrm{T}} \simeq 630 \times 2 \pi \mathrm{Hz}$. Lower panel parameters are as for figure 3 .

the frequency, it is characterised by a quite small amplitude $\omega_{2} \ll \bar{\omega}_{\mathrm{M}}(N)$, so it is surprising that driving the system so far off-resonance with the mechanical frequency is associated with a dramatic change in the output spectrum: the ratio $r \rightarrow 0$ for $N \approx 400$. In the quantum model, the underlying cause was shown to be (eg see Eq.(15)) to be a resonance between $\omega_{2}$ and the drive frequency, when $\omega_{2} \simeq 2 \omega_{\mathrm{d}}$. In the next section we introduce an analytical fast-cavity model which gives a different perspective to this suppression of one of the 'twin peaks'.

\section{Results: Further analysis of sideband asymmetry}

In this section we consider an alternative means to understand the sideband asymmetry in the hybrid trap. Although not central to the study of thermometry, it adds physical insight. 
Consider the linearised interaction Hamiltonian: $H_{\mathrm{OM}}^{\operatorname{lin}}=\hbar g(t) x(t) y_{\mathrm{a}}(t)$, where $y_{\mathrm{a}}(t) \equiv \hat{a}^{\dagger}(t)+$ $\hat{a}(t)$ is the amplitude quadrature of the cavity field.

The displacement $x(t)=\mathrm{X}_{\mathrm{M}}(t)+x_{0}(t)$ may be decomposed into a sum of a fast mechanical oscillation and a slow drift in the equilibrium position. The fast oscillation is $\mathrm{X}_{\mathrm{M}}(t) \simeq \mathrm{X}_{\mathrm{M}} \cos \Phi_{\mathrm{M}}(t)$ where $\mathrm{X}_{\mathrm{M}}$ is the variance of the thermal motion and the accumulated phase $\Phi_{\mathrm{M}}(t)=\int_{0}^{t} \omega_{\mathrm{M}}\left(t^{\prime}\right) d t^{\prime}=$ $\int_{0}^{t}\left(\bar{\omega}_{\mathrm{M}}+2 \omega_{2} \cos 2 \omega_{\mathrm{d}} t^{\prime}\right) d t^{\prime}$. Hence, we write:

$$
x(t)=X_{\mathrm{M}} \cos \left(\bar{\omega}_{\mathrm{M}}(N) t+\frac{\omega_{2}(N)}{\omega_{\mathrm{d}}} \sin 2 \omega_{\mathrm{d}} t\right)+X_{\mathrm{d}} \sin \omega_{\mathrm{d}} t,
$$

Substituting (16) and (22) into the interaction Hamiltonian we obtain:

$$
H_{\mathrm{OM}}^{\operatorname{lin}}=2 \hbar \bar{g} \sin \omega_{\mathrm{d}} t\left[X_{\mathrm{M}} \cos \left(\bar{\omega}_{\mathrm{M}}(N) t+\frac{\omega_{2}(N)}{\omega_{\mathrm{d}}} \sin 2 \omega_{\mathrm{d}} t\right)+X_{\mathrm{d}} \sin \omega_{\mathrm{d}} t\right] y_{\mathrm{a}}(t) .
$$

Expanding the expression with Bessel and trigonometric identities, and discarding $\bar{\omega}_{\mathrm{M}} \pm 3 \omega_{\mathrm{d}}$ terms,

$$
\begin{aligned}
& H_{\mathrm{OM}}^{\operatorname{lin}}=2 \hbar \bar{g} y_{\mathrm{a}}(t) X_{\mathrm{M}}\left\{\left[J_{0}(\phi)-J_{1}(\phi)\right] \cdot \sin \left(\bar{\omega}_{\mathrm{M}}(N)+\omega_{\mathrm{d}}\right) t\right. \\
& \left.+\left[J_{0}(\phi)+J_{1}(\phi)\right] \cdot \sin \left(\bar{\omega}_{\mathrm{M}}(N)-\omega_{\mathrm{d}}\right) t\right\},
\end{aligned}
$$

where $J_{i}$ is the $i$ th-order Bessel polynomial of the first kind parameterised by $\phi=\phi(N)=\frac{\omega_{2}(N)}{\omega_{d}}$. We then easily see that the optical field probes the split frequency $\bar{\omega}_{\mathrm{M}}(N) \pm \omega_{\mathrm{d}}$ with different weights. The initial phase of the Paul trap drive is irrelevant to the asymmetry; what matters is $g(t)$ and $\omega_{\mathrm{M}}(t)$ are antiphase, which naturally follows from $H_{\mathrm{OM}}^{\mathrm{lin}}$.

The equation shows that if $J_{0}(\phi)=J_{1}(\phi)$, the coupling to the $\bar{\omega}_{\mathrm{M}}(N)+\omega_{\mathrm{d}}$ frequency vanishes. Thus for this model one expects the ratio $r$ to be zero if $\frac{\omega_{2}(N)}{\omega_{\mathrm{d}}} \sim 1.4$, which is not too far from the prediction of the quantum model, $\frac{\omega_{2}(N)}{\omega_{\mathrm{d}}} \sim 2$.

We may also derive the split-sideband ratio using a fast-cavity picture. For $\kappa \gg \omega_{\mathrm{M}}$, the cavity field $a(t)$ instantaneously follows the mechanical motion; for optical trapping however, $a(t) \propto \cos ^{2} k x(t)$; light scattered from the cavity has a similar dependence. Substituting the form for $x(t)$ from Eq.22, the Fourier transform of $\cos ^{2} k x(t)$ may be used to calculate the cavity output PSD.

In general, sidebands of $\bar{\omega}_{\mathrm{M}}(N) \pm n \omega_{\mathrm{d}}, n=1,3,5, .$. will appear, as observed in figure 3 . With a more sophisticated detection, we may be able to resolve these higher-order sidebands in the experiments. For now, however, we are interested with the coefficients of $\bar{\omega}_{\mathrm{M}}(N) \pm \omega_{\mathrm{d}}$ and their ratio:

$$
r=\frac{J_{0}\left[X_{\mathrm{M}} J_{0}(\phi)\right] J_{1}\left[X_{\mathrm{M}} J_{1}(\phi)\right]-J_{0}\left[X_{\mathrm{M}} J_{1}(\phi)\right] J_{1}\left[X_{\mathrm{M}} J_{0}(\phi)\right]}{J_{0}\left[X_{\mathrm{M}} J_{0}(\phi)\right] J_{1}\left[X_{\mathrm{M}} J_{1}(\phi)\right]+J_{0}\left[X_{\mathrm{M}} J_{1}(\phi)\right] J_{1}\left[X_{\mathrm{M}} J_{0}(\phi)\right]} .
$$

With the simultaneous excursions in $g$ and $\omega_{\mathrm{M}}$, the optical field remains equally sensitive to the split-frequency $\bar{\omega}_{\mathrm{M}}(N) \pm \omega_{\mathrm{d}}$ only when $\phi(N)=\frac{\omega_{2}(N)}{\omega_{\mathrm{d}}}=0$; this happens at $N=0$. As $N$ increases and $\omega_{2}(N)$ becomes stronger, the twin-peak evolves into an asymmetric pair. In fact, one of the peaks completely vanishes when the condition $J_{0}(\phi)=J_{1}(\phi)$ is met, i.e. when $\phi(N) \approx 1.4$. We see this condition clearly from (25) as well. For the parameters in figure 3 , numerics indicate that complete cancellation happens at $N \approx 400$, while the fast-cavity model predicts $N \approx 362$. The quantum model, valid for both fast and slow cavities, gives an even closer estimate: $\omega_{2}(N) \approx 2 \omega_{\mathrm{d}}$ 
at $N \approx 419$. As with $\bar{\omega}_{\mathrm{M}}(N)$, accuracy could be improved by considering $\Delta$-modulation that corrects $|\bar{\alpha}|^{2}$.

The absence of $\Gamma_{\mathrm{M}}$ in (25) means the ratio persists even as the pressure is lowered. However, as we have not included quantum noises, the fast-cavity description is valid only in the thermal regime; the sideband ratio changes when quantum correlations become significant [24]. But, even if the quantum model may be extended to the ground-state, it is a linearised description. The fast-cavity model, while fully classical, includes all nonlinear behavior including the quadratic $x^{2}$-coupling that dominate low- $N$ dynamics. This is not predicted by the quantum model. In the weak cooling regime, only the fast-cavity model is able to describe the dynamics, allowing the ratio of $\omega_{\mathrm{M}} \pm \omega_{\mathrm{d}}$ to $2 \omega_{\mathrm{M}}$ to be obtained [22].

In the next section we outline how to do thermometry with a normalised cavity output using $\bar{g}(N)$ and $\bar{\omega}_{\mathrm{M}}(N)$ that we derived in section 3.

\section{Results: Split-sideband thermometry}

As illustrated in figure 2 and figure 3, the sidebands of the cavity output field in the case of a hybrid trap differ qualitatively from the standard case, with split-sideband structures seen both at low and high $N$. For low $N$ (weak cooling), $S_{\text {yout yout }}(\omega)$ has twin-peak pairs near $\omega= \pm \omega_{\mathrm{M}}$ and a further large peak at $\pm 2 \omega_{\mathrm{M}}$ due to $x^{2}$ coupling to the light. For higher $N$ (strong cooling), the split-sideband becomes asymmetric and the position-squared effects are strongly suppressed. As we are interested in strong cooling, we do not consider $x^{2}$ coupling here. A key question we test here is whether usual procedures for inferring temperature experimentally are still reliable for these non-standard output spectra.

In the canonical optomechanical case, the PSD of the position spectrum $S_{\mathrm{xx}}(\omega)$ may be deduced from the cavity output (in this case exemplified by the amplitude quadrature) from the approximate relation:

$$
S_{\text {yout }_{\text {out }}}(\omega) \simeq g^{2}|\eta(\omega)|^{2} S_{\mathrm{xx}}(\omega),
$$

where the optical susceptibility $\eta(\omega)=\chi_{\mathrm{O}}(\omega)-\chi_{\mathrm{O}}^{*}(-\omega)$ was defined in Sec 2.2. The expression may be deduced from (12), but neglecting the $\hat{Y}_{\text {in }}$ terms. In the limit of long measurement time, the Wiener-Khinchin theorem connects the Fourier transform of the autocorrelation function with the power spectral density: $S_{\mathrm{xx}}(\omega)=\left\langle|\hat{\mathrm{x}}(\omega)|^{2}\right\rangle$. The area under $S_{\mathrm{xx}}(\omega)$ then gives the variance $\left\langle|\hat{\mathrm{x}}|^{2}\right\rangle$ which the equipartition theorem relates to temperature [33]:

$$
\frac{1}{2 \pi} \int_{-\infty}^{+\infty} S_{\mathrm{xx}}(\omega) \mathrm{d} \omega=\left\langle|\hat{\mathrm{x}}|^{2}\right\rangle=\frac{k T}{m \omega_{\mathrm{M}}} .
$$

Using (26) and (27), the temperature can be extracted from a properly normalised cavity output:

$$
\frac{1}{2 \pi} \int_{-\infty}^{+\infty} \frac{S_{\text {yout }_{\text {out }}}(\omega)}{g^{2}|\eta(\omega)|^{2}} \mathrm{~d} \omega=\frac{k T}{m \omega_{\mathrm{M}}}
$$

Note that in the standard case, $S_{\mathrm{xx}}(\omega)$ and $S_{\text {youtyout }}(\omega)$ have very similar shapes and are peaked at $\omega_{\mathrm{M}}$. All the constant coupling parameter $g$ does is to rescale the area under $S_{\mathrm{y}_{\text {out }} \text { out }}(\omega)$. It 
is implicit that the above integrals are over the mechanical sidebands only. This is especially important in the numerics or near the quantum regime since $S_{\text {youtyout }}(\omega)$ may have a background arising from so-called imprecision noise, due to shot noise or classical laser noise. Near the quantum regime, the area of the sidebands is small and the background can contribute a significant error if the integral spans a frequency range larger than the sideband.

For hybrid trap experiments and simulations however, the equivalent expression to (26) is:

$$
S_{\text {yout } \text { Yout }}(\omega) \simeq \bar{g}^{2}|\eta(\omega)|^{2} S_{\mathrm{X}^{ \pm} \mathrm{X}^{ \pm}}(\omega),
$$

The optical field now probes the shifted displacement spectrum: $S_{\mathrm{X}^{ \pm} \mathrm{X}^{ \pm}}(\omega)=\left\langle\left|\hat{\mathrm{X}}^{ \pm}(\omega)\right|^{2}\right\rangle=$ $\left\langle\left|\hat{\mathrm{x}}\left(\omega+\omega_{\mathrm{d}}\right)-\hat{\mathrm{x}}\left(\omega-\omega_{\mathrm{d}}\right)\right|^{2}\right\rangle$.

Equation (29) was derived in [24] for arbitrary optomechanical coupling, but which we have explicitly related in Sec.3 $N$ in (17); hence $\bar{g} \equiv \bar{g}(N)$. In a manner similar to (27), we can extract the temperature from $S_{\mathrm{X}^{ \pm} \mathrm{X}^{ \pm}}(\omega)$ :

$$
\frac{1}{2 \pi} \int_{-\infty}^{+\infty} S_{\mathrm{X}^{ \pm} \mathrm{X}^{ \pm}}(\omega) \mathrm{d} \omega=\left\langle\left|\hat{\mathrm{X}}^{ \pm}\right|^{2}\right\rangle=\frac{k T}{m \omega_{\mathrm{M}}} .
$$

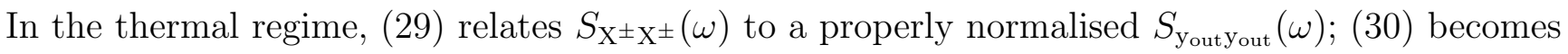

$$
\frac{1}{2 \pi} \int_{-\infty}^{+\infty} \frac{S_{\mathrm{y}_{\text {out }} \mathrm{Y}_{\text {out }}}(\omega)}{\bar{g}(N)^{2}|\eta(\omega)|^{2}} \mathrm{~d} \omega=\frac{k T}{m \omega_{\mathrm{M}}} .
$$

which relates the temperature to the split-sideband cavity output spectra, via an $N$-dependent normalisation.

In figure 4 we test (31). We obtain the temperature independently by calculating the area of $S_{\mathrm{xx}}(\omega)$ obtained from the full stochastic numerics; this is accessible to theory but not usually to experiments measuring homodyne or heterodyne cavity spectra. We compare this temperature with that obtained from a numerical simulation of $S_{\text {youtyout }}(\omega)$.

Additionally, we also estimate the temperature (in K) from the balance between heating and cooling rates. The dissipative part of the effective response function gives the optomechanical cooling rate [33]: $\Gamma_{\mathrm{opt}}(t)=g^{2}(t) \kappa\left[\mathcal{S}\left(\omega_{\mathrm{M}}\right)-\mathcal{S}\left(-\omega_{\mathrm{M}}\right)\right]$, where $\mathcal{S}\left(\omega_{\mathrm{M}}\right)=\left[\left(\Delta+\omega_{\mathrm{M}}\right)^{2}+\frac{\kappa^{2}}{4}\right]^{-1}[33]$. As it depends on $g^{2}(t)$, the cooling rate varies cyclically, reaching a maximum when the bead is at the linear region of the optical well. Knowing that $g, \omega_{\mathrm{M}}$, and $\Delta$ have $N$-dependent average values, we use (17), (20), and $\bar{\Delta}=\Delta+A$ to get the time-averaged optomechanical cooling rate:

$$
\Gamma_{\text {opt }}(N)=4 \bar{g}^{2}(N) \kappa\left\{\left[\left(\bar{\Delta}+\bar{\omega}_{\mathrm{M}}(N)\right)^{2}+\frac{\kappa^{2}}{4}\right]^{-1}-\left[\left(\bar{\Delta}-\bar{\omega}_{\mathrm{M}}(N)\right)^{2}+\frac{\kappa^{2}}{4}\right]^{-1}\right\} .
$$

An effective temperature can then be obtained:

$$
T \simeq 300 \frac{\Gamma_{\mathrm{M}}}{\Gamma_{\mathrm{opt}}(N)+\Gamma_{\mathrm{M}}} .
$$

For high $N$, the accuracy of (33) is limited by the small-angle approximations we used in deriving the shifted average parameters; nonetheless, figure 4 shows good agreement, at least for high $N$, between the three methods: time-averaging (33), from the area of the sidebands of $S_{\mathrm{xx}}(\omega)$ and of the normalised $S_{\text {youtyout }}(\omega)$. 
There are some evident discrepancies. For really low $N$, however, we see a discrepancy that can be explained by noting the cavity output spectra $S_{\text {youty }_{\text {out }}}(\omega)$ are dominated by the nonlinear $x^{2}$-coupling sidebands at $\omega \simeq \pm 2 \omega_{\mathrm{M}}$. This sideband is not present in $S_{x x}(\omega)$; this source of error could be improved by integrating only the area under the $\omega \sim \omega_{\mathrm{M}}$ sidebands; nevertheless, care is needed for $N \lesssim 100$.

In the important sideband resolved case of figure 4(a) which can lead to ground state cooling, there are increasing discrepancies with lower pressure as the sideband area becomes small; this is partly a numerical error which requires stronger averaging by the stochastic numerics; however, the systematic overestimate of temperature by $S_{\text {yout } \text { yout }}(\omega)$ is attributed to the contribution from the imprecision floor (from laser shot noise). With knowledge of $\omega_{\mathrm{M}}$ this can be improved by integrating strictly only over the frequency range under the sidebands; as the mean frequency changes with $N$, at present the numerics integrate over a much wider frequency range than is spanned by the sidebands.

For the case of the fast cavity, there is also a systematic over-estimation of the temperature from $S_{\text {youtyout }}(\omega)$ even at the highest pressures, where the sideband area is large and the imprecision noise contribution is negligible. We attribute this to the uncertainty in $g(N)$; The temperature depends on $(g(N))^{2}$ so is sensitive to this value; this may indicate that the analytically computed $g(N)$ in the fast cavity dynamics is underestimated by $20-30 \%$. There is also an extremely large discrepancy between the Langevin numerics and the optomechanical formula for low $N$; calculation of sideband area in this regime is numerically challenging: since $\Gamma_{\mathrm{M}} \propto P$, in this case, the cooling rates $\Gamma_{\text {opt }}(N) \rightarrow 0$ for low $N$ (e.g., for the experiments considered here, $\Gamma_{\mathrm{M}} \approx 0.11 \times 10^{4} P \mathrm{~s}^{-1}$ where the pressure $P$ is in mBar). The sidebands are thus extremely narrow so the numerical estimation of the area under the sidebands becomes very difficult.

Figure 4 also indicates the approximate point where $r \approx 0$ and a single peak (as opposed to a twin-peak structure) dominates the sideband structure of the cavity output. This provides a signature of high $N$ capture. The inferred value of $N$ represents a key source of uncertainty for experimental thermometry since $N$ is at present inferred from the sideband shape. Fortunately for $N \gg 100$ the variation of temperature on $N$ is sufficiently slow, that an uncertainty in $N \sim 10-50$ still allows a reasonable estimate of $g(N)$.

\section{Summary and Conclusions}

The use of Paul traps has opened the way for ground state cooling of nanoparticles in optical cavities, without the need for additional feedback cooling and at high vacuum. However, the resulting quantum noise spectra are atypical and complete understanding of the cavity output is essential not only for thermometry but also for any future sensing applications. The complex split-sideband structure potentially offer additional diagnostics of the dynamics including in the quantum regime [24], which further motivates such studies. In the present work we have analysed the $N$-dependence of the cavity output spectra, cooling dynamics, and optomechanical parameters. We show that the mechanical frequencies are slightly dependent on $N$ and shift to slightly lower 
values with increasing $N$. The shift is however quite predictable so easily calculated from either numerics or analytically for $N$ not too high.

In particular, asymmetries in the sidebands of the cavity output, arising from the effect of the ion trap drive and characterised by the ratio $r(N)$, were investigated. The $r=0$ point provides a distinctive feature which can be investigated in future experimental studies. From the quantum linearised dynamics, it is to predicted to occur if $\omega_{2}=2 \omega_{\mathrm{d}}$. We introduce a fast cavity model, which is valid only if $\kappa \gg \omega_{\mathrm{M}}$, but which gives further insight as it also produces an $r=0$ point. We have also investigated and compared temperatures extracted from the simulated cavity output spectra with those obtained from standard optomechanical cooling rate expressions, but using period-averaged parameters. We conclude that although the cavity output spectra have a complex sideband structure, temperatures may still be reliably inferred which are consistent with those calculated directly from the displacement spectra.

\section{APPENDIX: Measuring $\omega_{\mathrm{T}}^{2}$ and nanosphere charge $Q$.}

A Paul trap, in addition to the oscillating field with a drive frequency $\omega_{\mathrm{d}}$ may also include a static field $V^{\mathrm{DC}}(x, y, z) \frac{1}{2} m \omega_{\mathrm{DC}}^{2}\left(x^{2}+y^{2}-2 z^{2}\right)$.

The corresponding frequencies depend on the applied voltages $U_{\mathrm{AC}}$ and $U_{\mathrm{DC}}$ respectively; $\frac{1}{2} m \omega_{\mathrm{T}}^{2}=Q U_{\mathrm{AC}} / r_{0}^{2}$ while $\frac{1}{2} m \omega_{\mathrm{DC}}^{2}=Q U_{\mathrm{DC}} / r_{0}^{2}$ where $r_{0} \simeq 0.5 \mathrm{~mm}$ for the present study and $Q$ is the charge.

In the absence of a cavity, the $x, y, z$ motions are separable and are given by Mathieu equations,

$$
m \ddot{u}=\frac{m \omega_{\mathrm{d}}^{2}}{4}\left[a_{u}-2 q_{u} \cos \left(\omega_{\mathrm{d}} t\right)\right] u
$$

for $u=x, y, z$ and where $a_{\mathrm{x}, \mathrm{y}}=Q U_{\mathrm{DC}} /\left(m \omega_{\mathrm{d}}^{2} r_{0}^{2}\right)$ and

$$
q_{\mathrm{x}, \mathrm{y}}=4 Q U_{\mathrm{AC}} /\left(m \omega_{\mathrm{d}}^{2} r_{0}^{2}\right)
$$

while $a_{\mathrm{z}}=-a_{\mathrm{x}}$ and $q_{\mathrm{z}}=-2 q_{\mathrm{x}}$. In the present study, $U_{\mathrm{DC}}=0$ and no DC offset is applied.

Due to their importance in mass spectrometry, the Mathieu equations are well-studied (see [32] for a review). Since $\omega_{\mathrm{d}} \gg \omega_{\mathrm{T}}$, the motions are adiabatically separable into fast micromotion (oscillations at $\omega_{\mathrm{d}}$ ) and a slower secular motion. However, the secular motion is not characterized by $\omega_{\mathrm{T}}$; rather, it is characterized by a distinct so-called secular frequency; for modest $q_{u} \ll 0.9$, the secular frequency is given by:

$$
\omega_{\mathrm{s}}^{(u)} \simeq \frac{\omega_{\mathrm{d}}}{2} \sqrt{a_{u}+\frac{q_{u}^{2}}{2}}
$$

In the ion trap component of the hybrid trap, no static field is applied. However, provided transverse motions are of small amplitude relative to the beam waist, the optical mode provides an 

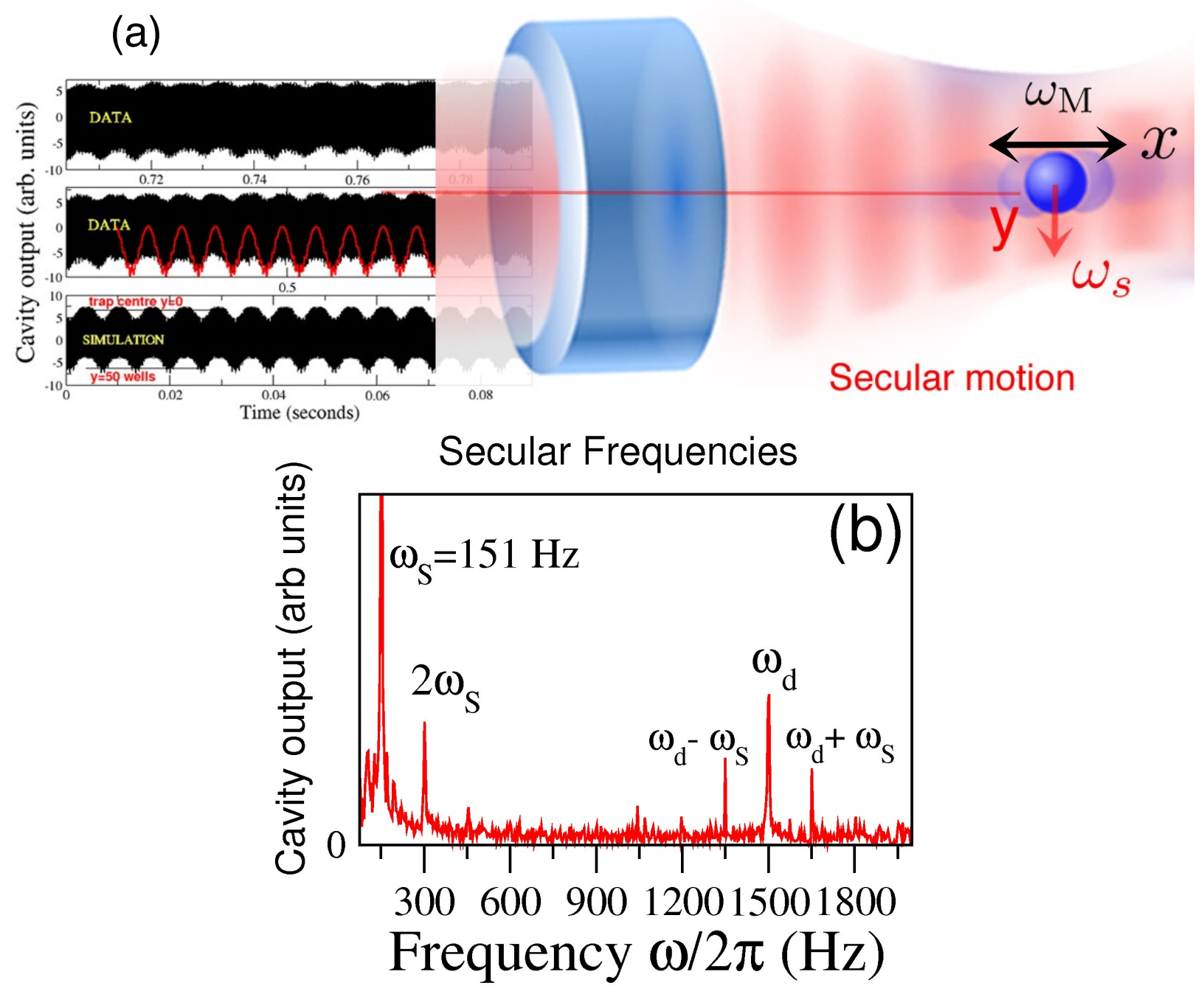

Figure 5. In order to calculate the optomechanical coupling $\bar{g}(N)$ and hence the cooling rates, an essential parameter is the Paul trap frequency $\omega_{\mathrm{T}}^{2}=\frac{2 Q V_{0}}{m r_{0}^{2}}$. This depends on the nanosphere charge $Q$ which can vary each time a new nanosphere is loaded into the hybrid trap. $\omega_{\mathrm{T}}^{2}$ is estimated by measuring the transverse (secular) frequencies of the trap, as well as the mechanical frequency, as explained in the Appendix. (a) Left panel illustrates a set of data where the amplitude of the $y$ motion is sufficiently large to visibly modulate the cavity output intensity (typically, however, the amplitude of secular motions is too small to be so apparent). The asymmetric nature of the trajectory in this case is even apparent: the upper, more rounded features correspond to the particle turning point nearer the centre $y=0$ of the optical beam; the lower, more pointed features correspond to a turning point further out (larger $y$ ) in the optical beam. (b) The low frequency part of the Fourier transforms of the cavity output data reveals $\omega_{\mathrm{s}}$ : in addition to peaks at $\omega_{\mathrm{s}}$ and its harmonics, there are also sidebands on the peak at the drive frequency.

approximate static potential. The Gaussian transverse confining potential $\mathcal{F}(y, z)=\exp \left[-2\left(y^{2}+\right.\right.$ $\left.z^{2}\right) / w^{2}$ ] represents an approximate harmonic potential for motions of amplitude $\ll w$. This introduces an effective DC parameter $a_{\mathrm{y}}=a_{\mathrm{z}}$ :

$$
a_{\mathrm{y}, \mathrm{z}}=\frac{16 \hbar A n}{m w^{2} \omega_{\mathrm{d}}^{2}},
$$


where $n=|\bar{\alpha}|^{2}$ is the photon number. For a non-ideal Paul trap, one must also define a trap efficiency $\epsilon$ :

$$
\omega_{\mathrm{T}}^{2}=2 Q \epsilon U_{\mathrm{AC}} / r_{0}^{2}=\frac{q}{2} \omega_{\mathrm{d}}^{2}
$$

The efficiency, which ranges from $0.1-1$ in different traps is the ratio between the voltage required to obtain a given secular frequency, relative to the ideal Paul trap, and absorbs uncertainties in the effective trap dimension parameter $r_{0}$. The value of $Q$ inferred from $\omega_{\mathrm{T}}$ or $q=Q \epsilon$ is an effective charge for a trap where the efficiency is not accurately known.

Substituting (37) in (36) and using (7), we get an effective secular frequency that is a sum of the original $\omega_{\mathrm{s}}^{2}$ with only $\mathrm{AC}$ drive, and a correction term proportional to $\bar{\omega}_{M}^{2}$ arising due to the photon field acting as a DC offset:

$$
\omega_{\mathrm{s}}^{2}(n)=\omega_{\mathrm{s}}^{2}(n=0)+\frac{2 \bar{\omega}_{\mathrm{M}}^{2}}{k^{2} w^{2}}
$$

where $\omega_{\mathrm{s}}^{2}(n=0)=\frac{\omega_{\mathrm{d}}^{2}}{4} \frac{q^{2}}{2}$, and $\bar{\omega}_{\mathrm{M}}=\bar{\omega}_{\mathrm{M}}(N=0) \approx \bar{\omega}_{\mathrm{M}}(N)$ for modest $N$. Through the readout of $\bar{\omega}_{\mathrm{M}}$ and the effective $\omega_{\mathrm{s}}(n)$ from the cavity output we can then infer $q$ using (39) and $\omega_{\mathrm{T}}^{2}$ using (38). 


\section{References}

[1] Yin Z Q, Geraci A A and Li T 2013 Int. J. Mod. Phys. B 271330018

[2] Romero-Isart O, Pflanzer A C, Blaser F, Kaltenbaek R, Kiesel N, Aspelmeyer M and Cirac J I 2011 Phys. Rev. Lett. 107020405

[3] Bateman J, Nimmrichter S, Hornberger K and Ulbricht H 2014 Nat. Commun. 54788

[4] Goldwater D, Paternostro M and Barker P F 2016 Phys. Rev. A 94010104

[5] Bahrami M, Paternostro M, Bassi A and Ulbricht H 2014 Phys. Rev. Lett. 112210404

[6] Geraci A A, Papp S B and Kitching J 2010 Phys. Rev. Lett. 105101101

[7] OConnell A D, Hofheinz M, Ansmann M, Bialczak R C, Lenander M, Lucero E, Neeley M, Sank D, Wang H, Weides M, Wenner J, Martinis J M and Cleland A N 2010 Nature 464 697-703

[8] Chan J, Alegre T P M, Safavi-Naeini A H, Hill J T, Krause A, Groeblacher S, Aspelmeyer M and Painter O 2011 Nature 47889

[9] Teufel J D, Donner T, Li D, Harlow J W, Allman M S, Cicak K, Sirois A J, Whittaker J D, Lehnert K W and Simmonds R W 2011 Nature 475359

[10] Chang D E, Regal C A, Papp S B, Wilson D J, Ye J, Painter O, Kimble H J and Zoller P 2010 Proc. Natl Acad. Sci. USA 1071005

[11] Barker P F and Shneider M N 2010 Phys. Rev. A. 81023826

[12] Romero-Isart O, Juan M L, Quidant R and Cirac J I 2010 New J. Phys. 12033015

[13] Monteiro T S, Millen J, Pender G A T, Marquardt F, Chang D and Barker P F 2013 New J. Phys. 15015001

[14] Kiesel N, Blaser F, Delić U, Grass D, Kaltenbaek R and Aspelmeyer M 2013 Proc. Natl Acad. Sci. USA 110 14180

[15] Millen J, Deesuwan T, Barker P F and Anders J 2014 Nat. Nanotechnol. 9425

[16] Ranjit G, Cunningham M, Casey K, Geraci A A 2016 Phys. Rev. A 93053801

[17] Mestres P, Berthelot J, Spasenovi M, Gieseler J, Novotny L and Quidant R 2015 Appl. Phys. Lett. 107151102

[18] Li T, Kheifets S and Raizen M G 2011 Nature Phys. 7527

[19] Gieseler J, Deutsch B, Quidant R and Novotny L 2012 Phys. Rev. Lett. 109103603

[20] Vovrosh J, Rashid M, Hempston D, Bateman J and Ulbricht H 2016 Controlling the Motion of a Nanoparticle Trapped in Vacuum arXiv:1603.02917.

[21] Jain V, Gieseler J, Moritz C, Dellago C, Quidant R and Novotny L 2016 Phys. Rev. Lett. 116243601

[22] Millen J, Fonseca P Z G, Mavrogordatos T, Monteiro T S and Barker P F (2015) Phys. Rev. Lett. 114 123602

[23] Fonseca P Z G, Aranas E B, Millen J, Monteiro T S, Barker P F 2016 Phys. Rev. Lett in press

[24] Aranas E B, Fonseca P Z G, Barker P F and Monteiro T S 2016 Toward detection of the quantum limit with slowly modulated optomechanics arXiv:1606.07377

[25] Ranjit G, Cris Montoya C and Geraci A A 2015 Phys. Rev. A 91013416

[26] Kuhn S, Kosloff A, Stickler B A, Patolsky F, Hornberger K, Arndt M and Millen J 2016 Full Rotational Control of Levitated Silicon Nanorods arXiv:1608.07315.

[27] Hoang T M, Ma Y, Ahn J, Bang J, Robicheaux F, Yin Z G and Li T 2016 Phys. Rev. Lett 117123604

[28] Delord T, Nicolas L, Schwab L, Htet G 2016 Electron spin resonance from NV centers in diamonds levitating in an ion trap arXiv:1605.02953

[29] Abbott B P et al. (LIGO Scientific Collaboration and Virgo Collaboration) 2016 Phys. Rev. Lett. 116061102

[30] Weinstein A J, Lei C U, Wollman E E, Suh J, Metelmann A, Clerk A A and Schwab K C 2014 Phys. Rev. X 4041003

[31] Peterson R W, Purdy T P, Kampel N S, Andrews R W, Yu P L, Lehnert K W and Regal C A 2016 Phys. Rev. Lett. 116063601

[32] March R E 1997 J. Mass Spectrom. 32351

[33] Aspelmeyer M, Kippenberg T J and Marquardt F 2014 Rev. Mod. Phys. 861391 